\title{
Spatial-temporal structure of seismicity of the North Tien Shan and its change under effect of high energy electromagnetic pulses
}

\author{
Nikolai T. Tarasov and Nadezhda V. Tarasova \\ Schmidt United Institute of Physics of the Earth, Russian Academy of Sciences, Moscow, Russia
}

\begin{abstract}
The effect of high-energy electromagnetic pulses emitted by a magnetohydrodynamic generator used as a source for deep electrical sounding of the crust on spatial-temporal structure of seismicity of the North Tien Shan is explored. Five-six years periodicity of changes in spatial distribution of seismicity was revealed. The effect of electromagnetic pulses increases the stability of the spatial distribution of seismicity over time and simultaneously speeds up cycles of its transformations, which develop on stabilization background. Increasing of seismic energy release after electromagnetic impacts is observed basically in most active zones. Periodic variation of efficiency of earthquakes triggering on the distance to the MHD-generator was detected. It was shown that electromagnetic pulses give rise to an appreciable increase in the rate of local earthquakes, occurring around 2-6 days after the pulses. Total earthquakes energy released after start-ups was by $2.03 \cdot 10^{15} \mathrm{~J}$ greater than the energy released before them. At the same time, the total energy transmitted by the MHD-generator was $1.1 \cdot 10^{9} \mathrm{~J}$, i.e. six orders of magnitude smaller. Consequently, the electromagnetic pulses initiated the release of the energy that had been stored in the crust due to activity of natural tectonic processes in the form of comparatively small earthquakes, which leads to an additional release of tectonic stresses.
\end{abstract}

Key words earthquake - initiated - seismicity electromagnetic - pulse

\section{Introduction}

The Bishkek site is situated in the junction area of North Tien Shan and Chuya Basin structures. Its southern part consists of Paleozoic crystalline rocks and the northern, of Mesozoic/ Cenozoic sedimentary deposits. The Tien Shan

Mailing address: Dr. Nikolai T. Tarasov, Schmidt United Institute of Physics of the Earth, Russian Academy of Sciences, ul. Bolshaya Gruzinskaya 10, D-242, GSP-5, 123995 Moscow, Russia; e-mail: tarasov@ifz.ru is a highly seismic region of the Earth. The north Tien Shan earthquake-generating zone, where the Bishkek site is situated, contains deep crustal faults. Three earthquakes with magnitudes great er then 8.0 occurred there during the past 100 years.

Deep electrical sounding of the crust was carried out at the Bishkek test site of the Institute of High Temperatures RAS in 1983 to 1990. The source of energy was the MHD-generator, and load was electrical dipole of $0.4 \Omega$ resistivity, whose electrodes were $4.5 \mathrm{~km}$ apart. When the generator was put into operation, the load current was $0.28-2.8 \mathrm{kA}$, the sounding pulses had durations of 1.7 to $12.1 \mathrm{~s}$, the energy being mostly in the range 1.2-23.1 MJ. The dipole was installed within those Paleozoic crystalline structures in the north Tien Shan $\left(42.69^{\circ} \mathrm{N}, 74.68^{\circ} \mathrm{E}\right)$, which 
were adjacent to the boundary of Mesozoic/ Cenozoic sedimentary deposits in the Chuya Basin. During the time of the MHD operation, 114 start-ups were carried out. A detailed description of the MHD equipment and the sounding procedure can be found in Volykhin et al. (1993).

The observation area was within coordinates $41.0^{\circ}$ to $45.5^{\circ} \mathrm{N}$ and $74.0^{\circ}$ to $81.5^{\circ} \mathrm{E}$. By the criterion of geological structure, the area can roughly be divided by latitude $42.9^{\circ}$ into a southern part (the Paleozoic features of North Tien Shan) and a northern part (Mesozoic/Cenozoic sedimentary deposits). Analysis of seismicity of the observation area was based on the earthquake catalog for the North Tien Shan and adjacent areas of N. Mikhailova containing earthquake data for the period from 1975 to 1996 (Mikhailova, 1990). The catalog contained data on 14114 earthquakes with magnitudes $M_{L}$ from 0 to 6.2. The recurrence diagram based on all events is linear in the $M_{L}$ ranging from 2.2 to 6.1.

The trigged seismicity was identified upon the background of the natural variation by coherent summation of earthquakes in time windows of \pm 20 days about the time of each of 114 MHD-generator start-ups (i.e. we superposed all 114 windows in time). For this purpose, selection of earthquakes was made for each of 114 MHD-generator runs in the interval $\left(t_{j}+\right.$ $-20, t_{j}+20$ ), where $t_{j}$ is absolute times (in days) of the $j$ th MHD-generator start-up. Absolute times $T_{i}$ of all selected earthquakes (more than 4000) were replaced by the times $t_{i j}=T_{i}+$ $-t_{j}$ measured from the time of the corresponding start up (here $i$ is number of earthquake in the selection). Obtained selection was used to find $N_{t}$, which is the number of events occurring in the area during each day within time windows, i.e. those with $t-0.5 \leq t_{i j} \leq t+0.5$ for all integer $t$ between -20 and 20 days.

Values $N_{t}$ with $t>0$ were analyzed to study the effect of electromagnetic pulses of the MHDgenerator on seismicity over time. The events occurring during 20 days before the start-up were used to estimate the natural seismicity rate immediately before MHD-generator runs. We calculated background level as averaged daily number of events and estimated the $99 \%$ confidence interval over all $N_{t}$ with $t<0$. For investigation of total seismic energy changes after MHD-generator runs, we calculated the ratio of the sums of the energy of earthquakes with $t_{i j}>0$ and $t_{i j}<0$, respectively, using the relationship for earthquake energy estimation presented in Rautian et al. (1981). Note, this technique allows one to increase the relation of signal/noise $\sqrt{n}$ times, where $n$ is the number of the superposed time windows. This brings the relation up to 10.7 when 114 start ups of the MHD-generator are used.

For study of spatial variations of total seismic energy changes after the MHD-generator runs, the observation area was divided into $1000 \mathrm{~km}^{2}$ cells of the grid. The ratio of the sums of the energy of earthquakes with $t_{i j}>0$ and $t_{i j}<0$, respectively, was calculated for each cell. The map of distribution energy ratios was created using all cells with non-zero earthquake number. A similar technique was used for creation of the map of the flow of total seismic energy over the area, but the total catalog was processed for this purpose instead of the selection made.

For a quantitative estimation of variations of a spatial distribution of the earthquakes over time, the spatial correlation of earthquakes density for each two contiguous years $r_{k}$ as a function of the time from 1975 to 1995 was calculated. To do this, the region of observations was divided into square $1000 \mathrm{~km}^{2}$ cells of the grid too. $n_{i j k}$ and $n_{i j k+1}$ are numbers of the earthquakes occurred inside each cell for $k$ th and for $k+1$ th years, respectively, were calculated for all $k$ varying from 75 to 95 year. The indices $i, j$ denote the numbers of a grid cell for latitude and longitude. They are related with geographical coordinates $X, Y$ as

$$
i=\operatorname{Int}\left(X-X_{0}\right) / d X, \quad j=\operatorname{Int}\left(Y-Y_{0}\right) / d Y
$$

where $d X, d Y$ are the length of sides of the cell on axes $X, Y$ in degrees, and $X_{0}=41^{\circ} \mathrm{N}$ and $Y_{0}=$ $=74^{\circ} \mathrm{E}$ are latitude of southern and longitude of western boundaries of the observation area. The function of spatial-temporal correlation of seismicity $r_{k}$ was evaluated for all $k=75,76, \ldots, 95$ using the expression

$$
r_{k}=\sqrt{\frac{C_{k, k+1}^{2}}{S_{k} \cdot S_{k+1}}}
$$


where

$$
\begin{aligned}
& C_{k, k+1}=\sum_{i=1}^{N} \sum_{j=1}^{M} n_{i j k} \cdot n_{i j k+1}-\frac{1}{N \cdot M} \cdot \\
& \left(\sum_{i=1}^{N} \sum_{j=1}^{M} n_{i j k} \cdot \sum_{i=1}^{N} \sum_{j=1}^{M} n_{i j k+1}\right) \\
& S_{k}=\sum_{i=1}^{N} \sum_{j=1}^{M}\left(n_{i j k}\right)^{2}-\frac{1}{N \cdot M}\left(\sum_{i=1}^{N} \sum_{j=1}^{M} n_{i j k}\right)^{2} \\
& S_{k+1}=\sum_{i=1}^{N} \sum_{j=1}^{M}\left(n_{i j k+1}\right)^{2}-\frac{1}{N \cdot M}\left(\sum_{i=1}^{N} \sum_{j=1}^{M} n_{i j k+1}\right)^{2}
\end{aligned}
$$

$N$ is number of cells along the axis $X$, and $M$ is number of cells on the axis $Y$.

\section{Results}

First, we examined the time variation in seismicity after the MHD-generator runs. Figure 1a presents the distribution of daily rates of earthquakes $N_{t}$ for the entire area before and after MHD-generator runs. The values of $N_{t}$ are seen to be appreciably higher one day after the startup than before it. The maximum rate occurred on the second day after start-up with amplitude nearly 1.7 above background level. When only earthquakes in the north part are considered, no significant changes in $N_{t}$ related to MHD-generator runs can be detected (fig. 1b), whereas a well-pronounced maximum can be seen in the south (fig. 1c) occurring on the second day after electromagnetic action, nearly 2.2 above the mean background, to be followed by a gradual falloff in $N_{t}$ during a few days.

The triggering effect of electromagnetic pulses of MHD-generator was most active in the upper $5 \mathrm{~km}$ layer. Figure $1 \mathrm{~d}$ shows the distribution of the earthquakes occurring within the layer in the south part of the area. It is seen that during the first day after MHD-generator runs, the $N_{t}$ values remain at the background level and then a strong activization of the regime occurs that goes on for about 5 days. During this period, $N_{t}$ exceeds background level 2.7 times. The maximum is observed on the second day after MHD-generator start-up. Its amplitude exceeds 99\% confidence level.

The above results show that high energy electromagnetic pulses emitted by MHD-generator give rise to an appreciable increase in the rate of local earthquakes, occurring about 2-6 days after the pulses. It confirmed the initiating effect of action by electromagnetic pulses on the seismicity previously revealed (Tarasov, 1997; Tarasov et al., 1999) in the Garm and Bishkek areas.

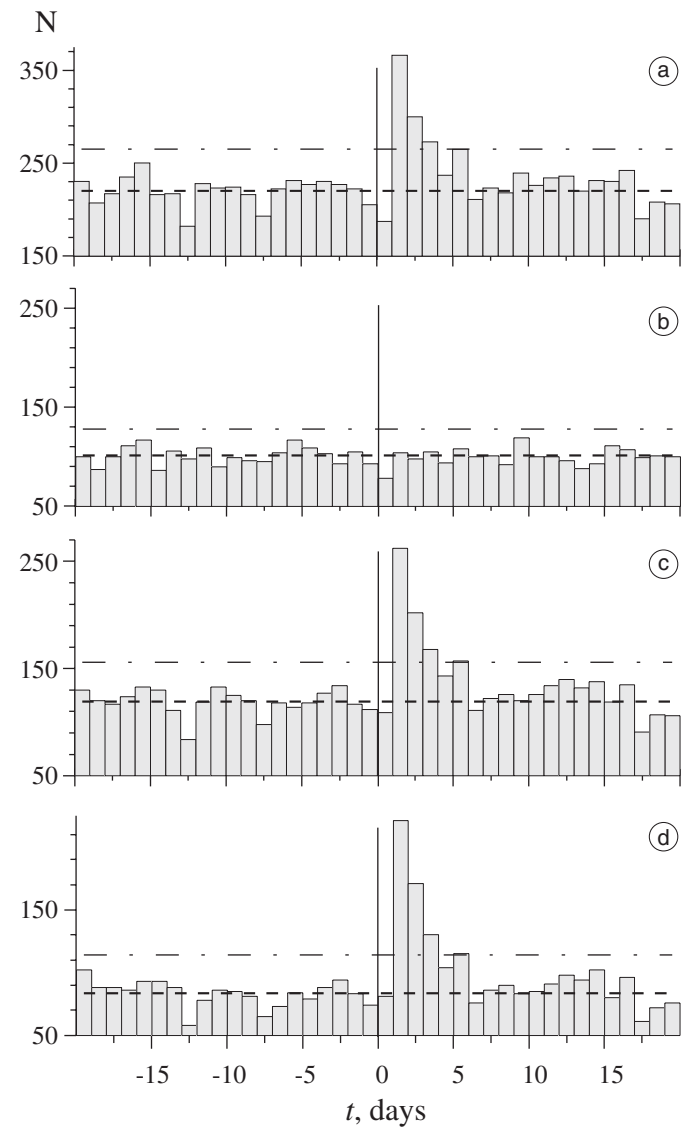

Fig. 1a-d. Daily number of earthquakes in the area of the Northern Tien Shan and adjacent territories before $(t<0)$ and after $(t>0)$ the MHD-generator runs on the Bishkek test site plotted against time: a) for the entire area, b) its northern and (c) southern (North Tien Shan) part, d) events occurring in the upper $5 \mathrm{~km}$ layer of the southern part of the region. The vertical axis shows the daily rate of earthquakes; horizontal axis shows the time measured from the MHD start-ups (in days). The dashed lines indicate a mean background level (lower lines) and 99\% confidence interval (upper lines). 


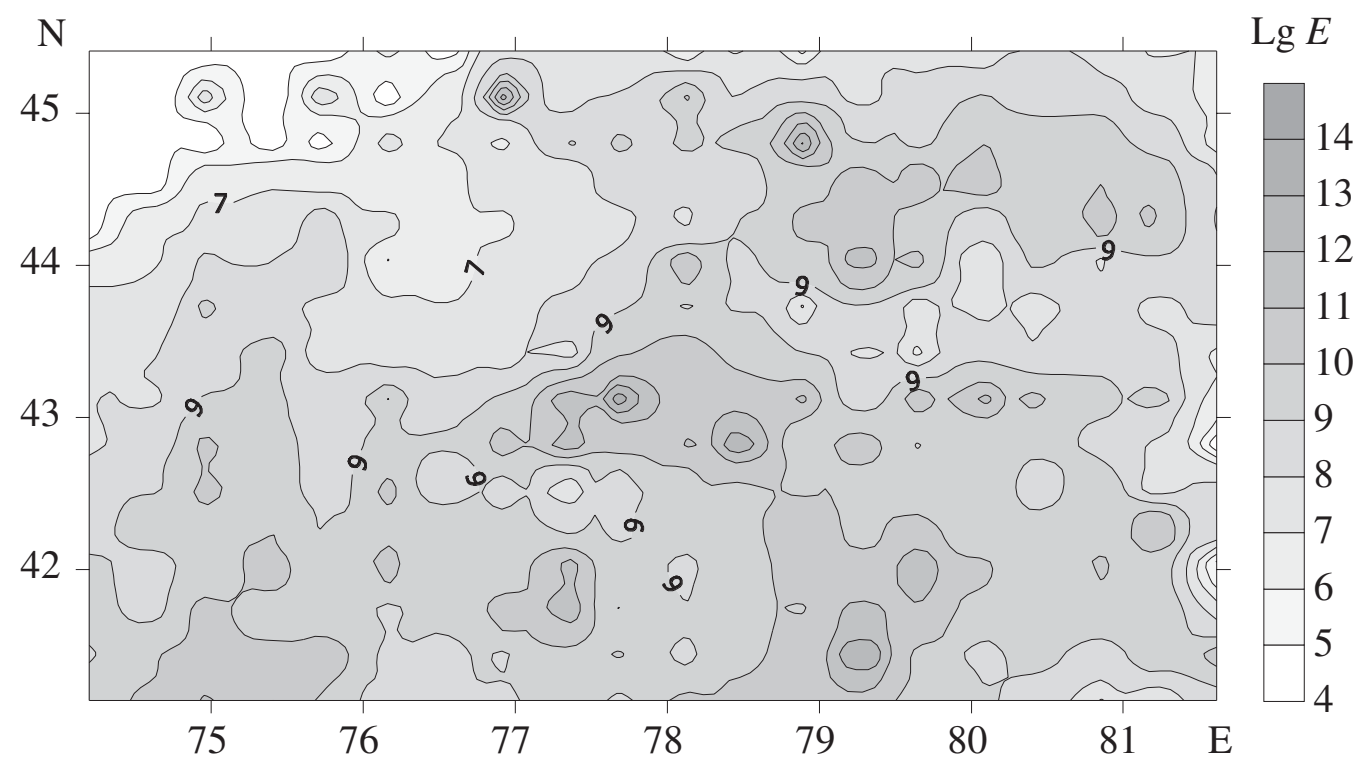

Fig. 2. Map of the flow of total seismic energy of earthquakes in Northern Tien Shan and adjacent territories obtained using all earthquakes of the catalog for $1000 \mathrm{~km}^{2}$ cells of grid. The values of energy are reduced to the flow for 1 year.

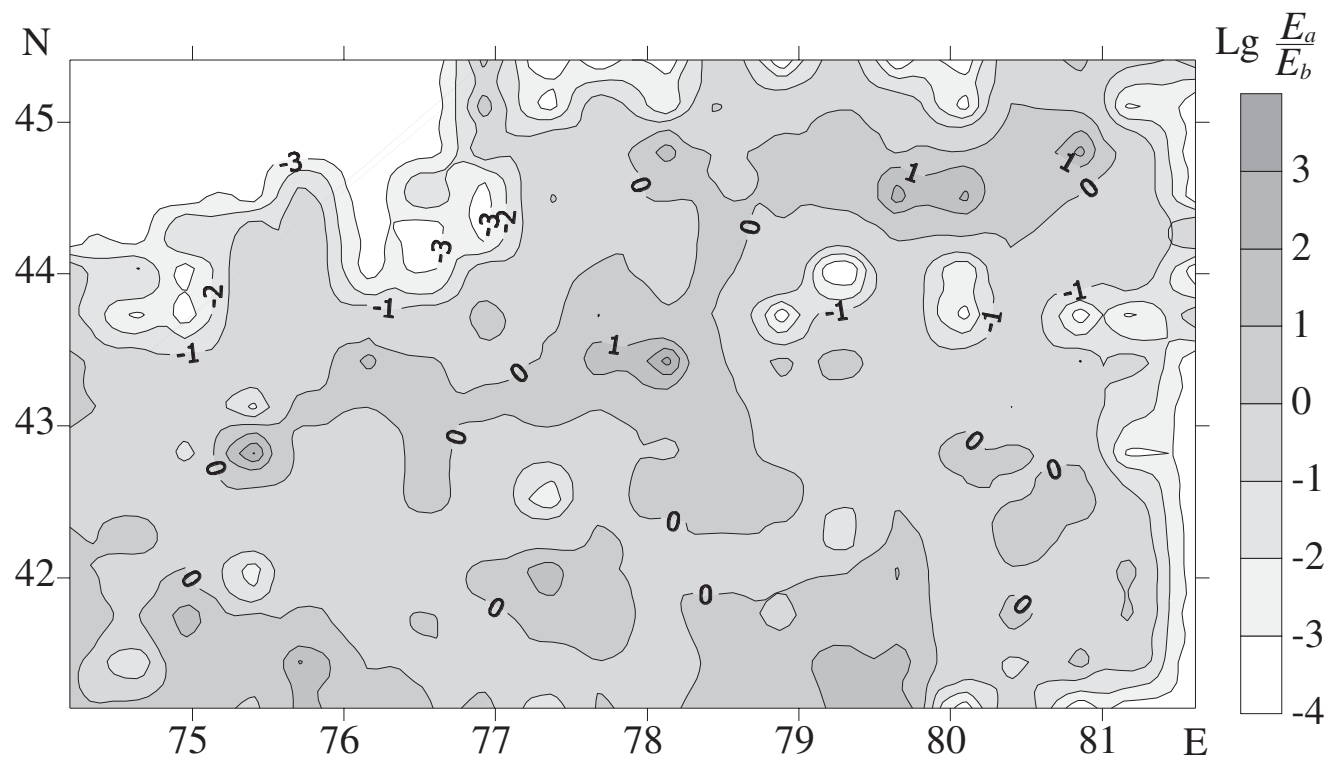

Fig. 3. Map of total seismic energy ratio of earthquakes in Northern Tien Shan area and adjacent territories for 20 days after and 20 days before MHD-generator runs. 
On the one hand it can be assumed that this effect results from the extra energy loaded to the Earth by electromagnetic pulses, which is radiated in the form of earthquakes. Another explanation is that sounding pulses initiated the release of energy stored in the crust due to natural geodynamic processes. To test these assumptions, we estimated the total seismic energy released during 20 days before all MHDgenerator start-ups $E_{b}$, total seismic energy released during the same period after the start-ups $E_{a}$, and the post-start-up increase in total seismic energy release $E_{a}-E_{b}$. These estimates were based on all earthquakes of the area, on events occurring in the north and south, regardless of their focal depths, and the events from the upper $5 \mathrm{~km}$ layer.

An analysis of the obtained values shows that the total earthquake energy released in the area during 20 days after all start-ups was by $2.03 \cdot 10^{15} \mathrm{~J}$ greater than the energy released for a period of the same duration before them. Most of the energy release was due to the earthquakes occurring in the upper $5 \mathrm{~km}$ layer in the southern part of the area, which belongs to the crystalline rocks of the North Tien Shan. At the same time, the total energy transmitted by MHD-generator to the radiating dipole for all start-ups was $1.1 \cdot 10^{9} \mathrm{~J}$, i.e. six orders of magnitude smaller. Consequently, the high energy electromagnetic pulses radiated by the MHDgenerator initiated the release of the energy that had been stored in the crust due to other sources.

The mean energy of initiated earthquakes per one start-up was $2.2 \cdot 10^{13} \mathrm{~J}$. Tarasov (1997) investigated the initiating effect of MHD-generator pulses on the seismicity of the Garm area in Tajikistan and concluded that the average increase in total energy release through local seismicity was $1.1 \cdot 10^{12} \mathrm{~J}$ per start-up. However it should be kept in mind that Garm site has an area much smaller than our area under study. When the estimates of energy were divided by the values of respective areas, the extra seismic energy release was $7.3 \cdot 10^{7} \mathrm{~J} / \mathrm{km}^{2}$ per start-up in the Bishkek site and $6.6 \cdot 10^{7} \mathrm{~J} / \mathrm{km}^{2}$ per start-up in the Garm site. These similar values indicate approximately similar responses of the Earth to electromagnetic pulses in different areas.
Figure 2 presents the map of the flow of total seismic energy of earthquakes of North Tien Shan and area of adjacent territories. The density of seismic energy flow is seen to vary widely over the area. There are several zones of high energy flow in the middle and south, while energy flow density in the northwest is significantly lower.

Figure 3 shows the spatial variations in the changes of total seismic energy after MHDgenerator start-ups. The map demonstrates that seismic energy release increasing takes place within a number relatively small zones located predominantly in southern and central parts of the observation area. One can see some correlation of maps in figs. 2 and 3. However, these maps are not identical. Therefore, electromagnetic effects cause the increase in seismic energy release mainly in most active zones. Nevertheless a triggering effect is determined not only by a level of seismic activity, but also by other phenomena.

Using this map, it is difficult to recognize any regular dependence of efficiency of trigger effects of electromagnetic pulses on the distance to location of the MHD-generator $\left(42.69^{\circ} \mathrm{N}, 74.68^{\circ} \mathrm{E}\right)$. For detailed analysis, we calculated average $E_{a} / E_{b}$ as function of the distance from location of the MHD-generator to epicenters of earthquakes, irrespective of azimuths. Using all MHD-generator runs, the summary seismic energy of earthquakes which occurred 20 days before $\left(E_{b}\right)$ and 20 days after $\left(E_{a}\right)$ electrical soundings were calculated for ring bands bounded by radii $R$ and $R+50 \mathrm{~km}$ with the center in the site of location of the MHD-generator. $E_{b}$ and $E_{a}$ were computed in the distance range from 0 up to $600 \mathrm{~km}$ with 50 $\mathrm{km}$ step.

Shown in fig. 4 , the dependence of $E_{b}$ on the distance to MHD-generator represents a natural change of the flow of seismic energy in the observational region. Similar dependence of $E_{a}$ shows summary effect of natural changes and trigged seismicity. Note, relatively low level of $E_{b}$ is observed up to distances of $\sim 200 \mathrm{~km}$. One can see in the figure that the total seismic energy before MHD-generator runs was higher than after them for distances $R<110 \mathrm{~km}$. Then, the interval where $E_{a}$ is greater than $E_{b}$ is observed. 


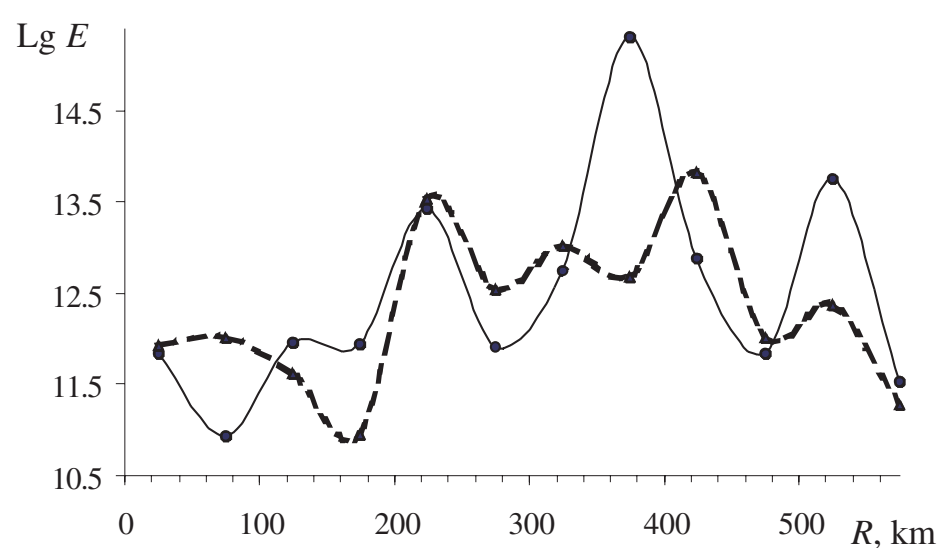

Fig. 4. The total seismic energy of earthquakes of the North Tien Shan and adjacent areas occurring before $E_{b}$ (dashed line) and after $E_{a}$ (solid line) 114 MHD-generator start-ups as a function of the distance to location of the MHD-generator on Bishkek test-site. The values $E_{a}$ and $E_{b}$ are plotted on a logarithmic scale.

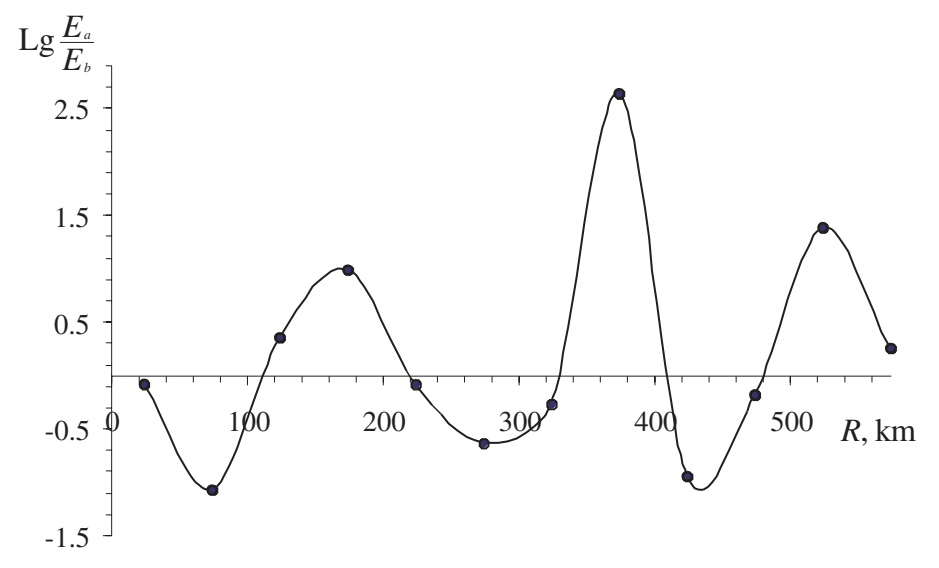

Fig. 5. The ratio $E_{a} / E_{b}$ of the total seismic energy of earthquakes of the North Tien Shan and adjacent areas occurring after and before $114 \mathrm{MHD}$-generator start-ups as a function of the distance to location of the MHDgenerator on Bishkek test-site. The values $E_{a} / E_{b}$ are plotted on a logarithmic scale.

Further, the position of curves relative to one another changes, and so on.

More clearly it is possible to see in fig. 5 where the dependence of $\operatorname{Lg}\left(E_{a} / E_{b}\right)$ being the ratio of the total seismic energy of earthquakes after and before MHD-generator start-ups on the distance is presented. The values $\operatorname{Lg}\left(E_{a} / E_{b}\right)$ change the sign periodically through every 80 $110 \mathrm{~km}$. Hence, bands where the electrical ef- fects increases the flow of seismic energy and bands where the electrical effects diminishes the seismic flow, alternate periodically with increasing the distance to the MHD-generator.

It is interesting that $\operatorname{Lg}\left(E_{a} / E_{b}\right)<0$ at the short distance (less than $100 \mathrm{~km}$ ). Therefore, the electromagnetic pulses diminish seismic activity inside near the field area of the electrical dipole. At distances of $110-220 \mathrm{~km}$, the seismici- 


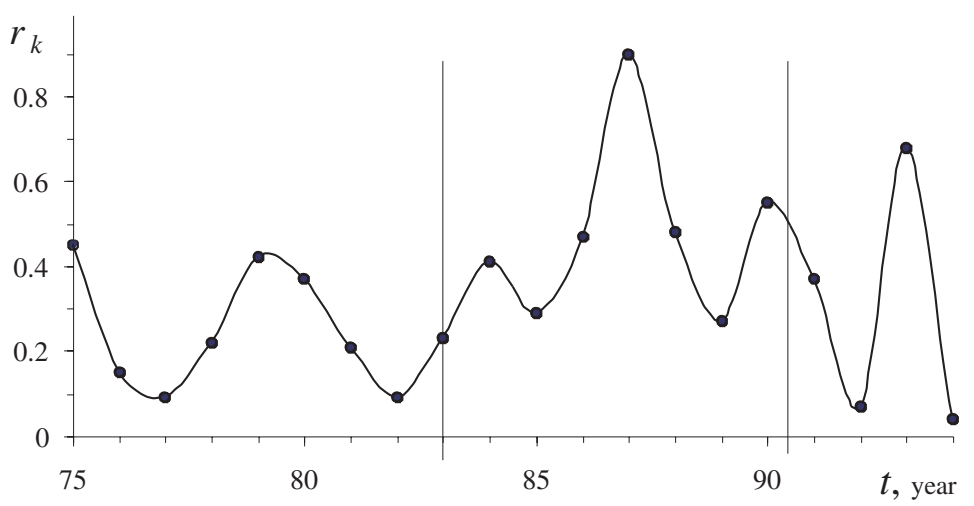

Fig. 6. Spatial-temporal correlation of density of earthquakes of the North Tien Shan and adjacent areas as function of time. The vertical axis indicates the values of a correlation function $r_{k}$; the horizontal axis indicates time in years. The vertical points are time of beginning and termination of a series of MHD-generator start-ups on the Bishkek test-site.

ty increases after MHD-generator start-ups, further, the curve goes to the negative domain again, etc. As a whole, the sign-variable dependence is observed, whose amplitudes increase up to a distance of $375 \mathrm{~km}$ to electrical dipole of the MHD-generator and, then, the amplitudes begin to decrease. It is important to note that the amplitudes of positive half-periods of the dependence are higher than the amplitudes of negative half-periods. This confirms that the effect of the electromagnetic pulses causes substantial growth of seismic energy of the region in the whole.

For a quantitative estimation of variations of a spatial distribution of the earthquakes over time, the spatial correlation $\left(r_{k}\right)$ of earthquakes density for each two contiguous years as function of the time from 1975 to 1995 year was calculated using the technique discussed in the first part of the paper. This function is presented in fig. 6. First of all, it is interesting that for the period of 19751983 the periodic changes of the function are clearly observed. For this time interval, value $r_{k}$ has rather low values. The correlation regularly varies from 0.1 to 0.45 with a period of 5-6 years. It shows that there are periods of «fast» changes in spatial distribution of seismicity, which are replaced by periods of its relative stability every other 2.5-3 years and vice versa.
One can clearly see in fig. 6 that the function abruptly changes the shape since 1983 . The 5-6 year periods of variations in correlation are changed by periods of 3 year duration. At the same time, a significant increase in correlation factors $r_{k}$ up to 0.3-0.9 is observed. Thus, the spatial distribution of earthquakes becomes more stable after 1983. As the time of change in frequency of periodicity and in increase in values $r_{k}$ coincides with the beginning of experiments with MHD-generator, it is possible to assume that the action of electromagnetic pulses causes an increase in stability of spatial distribution of seismicity over time and simultaneously speeds up cycles of its transformations, which develop on stabilization background.

Interestingly, the variation of values of fractal dimension shows that the earthquakes temporal distribution becomes more random for time period of experiments with the MHD-generator (from 1983 to 1990) (Chelidze et al., 2002).

Figure 7 shows a similar dependence based on the selection of earthquakes occurring in the upper $5 \mathrm{~km}$ layer of the crust. Just at the beginning of observations time interval, the rather high values of a correlation function are observed on the curve. Further, within 6 years from 1977 to 1983, the values of $r_{k}$ do not exceed 0.23 . At the beginning of experiments with 


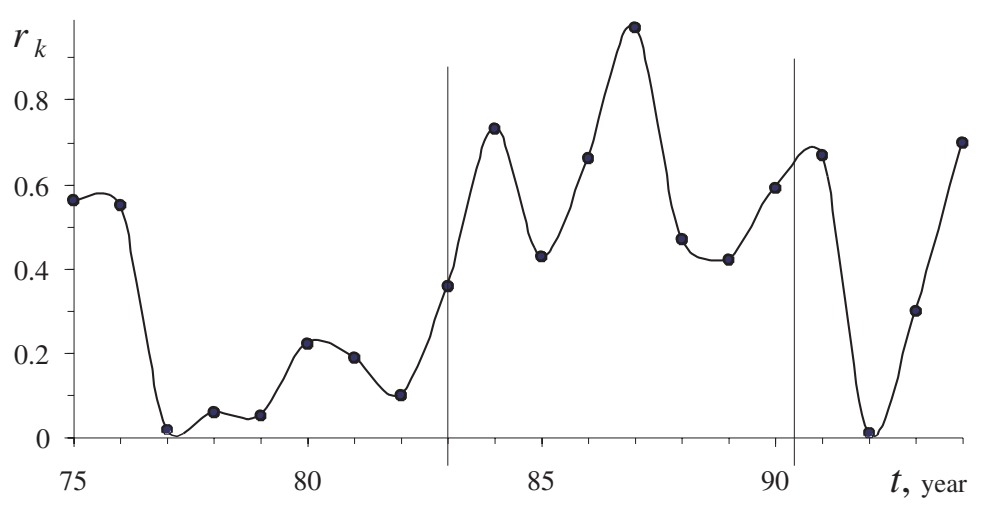

Fig. 7. Function of a spatial-temporal correlation of density of earthquakes occurringin the upper 5 km layer of crust of the North Tien Shan and adjacent areas. The vertical axis indicates the values of a correlation function $r_{k}$; the horizontal axis indicates time in years. The vertical points are the time of beginning and termination of a series of MHD-generator start-ups on the Bishkek test-site.

the MHD-generator, the level of spatial-temporal correlation sharply increases. Thus, in 1983, $r_{k}$ reaches 0.35 , and, further, the values oscillate within the range 0.42-0.97. The 3-4 year periodicity is observed for this time again. These results confirm that the most appreciable changes in spatial-temporal structure of seismicity after the beginning of experiments with a MHD-generator are observed in the upper $5 \mathrm{~km}$ layer.

Previously, we showed that the most intensive increase in total seismic energy of earthquakes after MHD-generator start-ups was observed at the same depths not only in the North Tien Shan, but also in the Garm region of Tadjikistan (Tarasov, 1997; Tarasov et al., 1999). Because the initiating action was most effective in the depth range $0-5 \mathrm{~km}$ which is the hydrostatic zone of the crust containing free water that fills cracks, pores and cavities, it was concluded that fluids are largely responsible for the initiation of seismicity by electromagnetic pulses. The presence of fluids in rocks gives rise to the seismoelectric and electroseismic effects.

To test this assumption, we studied the changes in efficiency of the earthquakes triggering by electromagnetic pulses depending on a level of atmospheric precipitates. First, the values $E_{a} / E_{b}$ obtained on each of 114 start-ups were compared to an average level of precipi- tates before each of them. To average the precipitate level before the start-ups, the time windows of different duration from 1 to 20 day were applied. However, a significant correlation between the flow of seismic energy and level of precipitates was not found.

Comparison of long-period changes of $E_{a} / E_{b}$ with average annual level of precipitates was more successful. To do this, the values of average annual level of precipitates were calculated for each year of experiments. For the same period, we computed the ratios $E_{a} / E_{b}$ of total seismic energy of the earthquakes which occurred in layer $0-5 \mathrm{~km}$ after and before the MHD-generator start-ups within each year. As the intervals between start-ups varied for different years, the time windows for $E_{a} / E_{b}$ calculation were limited by 5 days. Tarasov et al. (1999) have shown that it reduces the noise level which arises because of partial superposition of time windows of different start-ups when they are executed too often.

Figure 8 represents the variations $E_{a} / E_{b}$ depending on time for 1983-1990 years. The changes in the annual level of atmospheric precipitation in Bishkek are shown for the same period in the fig. 9. One can see that these curves are correlated. The correlation factor in this case was equal to 0.845 , though this esti- 


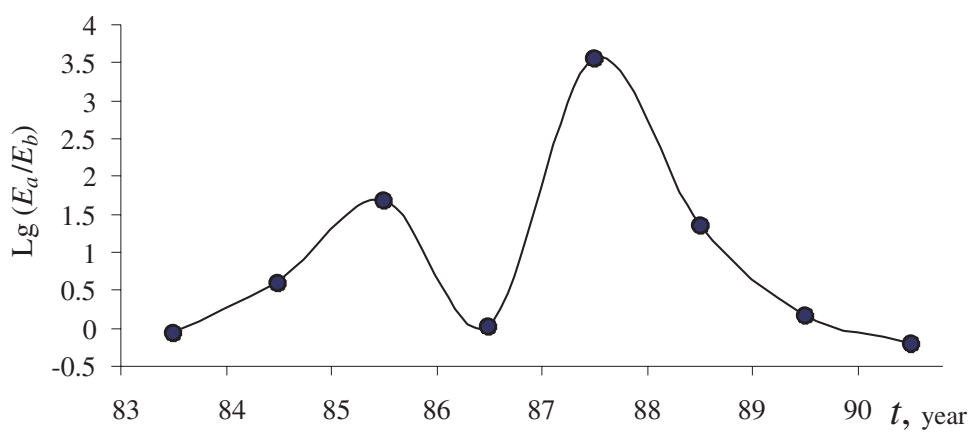

Fig. 8. Average annual values of $E_{a} / E_{b}$ being the ratio of total seismic energy of earthquakes of the North Tien Shan occurring 5 days after and 5 days before MHD-generator start-ups in the upper $5 \mathrm{~km}$ layer as a function of time. The values $E_{a} / E_{b}$ are plotted on a logarithmic scale.

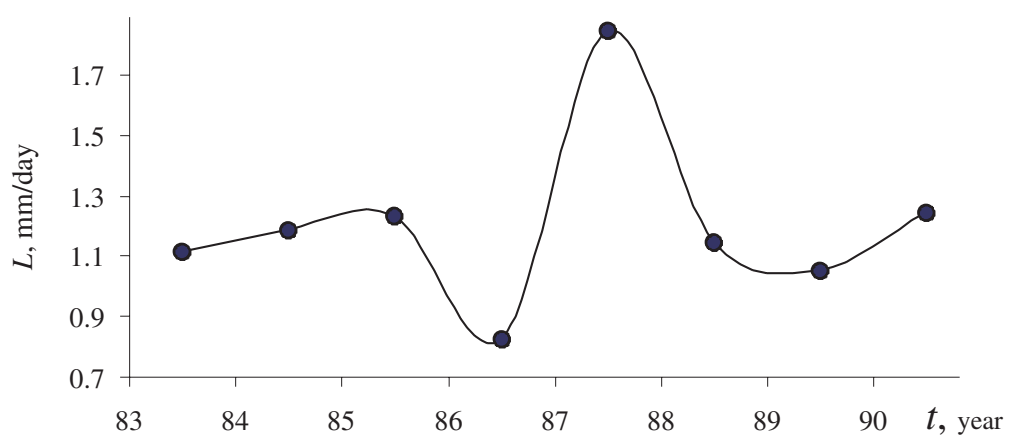

Fig. 9. Averaged annual level of atmospheric precipitation in Bishkek as a function of the time.

mation should be assumed as preliminary, because we have only very small statistics.

More clearly it could be seen in fig. 10 where points represents the ratio of total seismic energy after and before all MHD-generator start-ups for each of 8 years as a function of the annual level of precipitates for the related year. The fitted line obtained by the mean square method is also given. It is possible to see the values $E_{a} / E_{b}$ increase depending on the level of precipitates. These results confirm the assumption that fluid is largely responsible for initiation of earthquakes by electromagnetic pulses.

The dependence of efficiency of earthquakes triggering by electromagnetic pulses at a hypocenter depth shows that this can depend on pulse duration, as this parameter determines the thickness of the skin layer. Figure 11 represents $E_{a} / E_{b}$ as a function of sounding pulse duration. To calculate the diagram, all start-ups of the MHD-generator were divided into a number of subsequences with respect to pulse duration. The total seismic energy of the earthquakes that occurred before and after pulses of some duration was evaluated using all start-ups of MHD-generator included in the relevant subsequence.

In the figure we do not observe any regular increase or decrease in efficiency of electromagnetic effects with the pulse duration. One can see that the dependence has sign-variable character. The shortest (less than $1.5 \mathrm{~s}$ ) and longest (more than $12 \mathrm{~s}$ ) pulses cause a decrease in total 


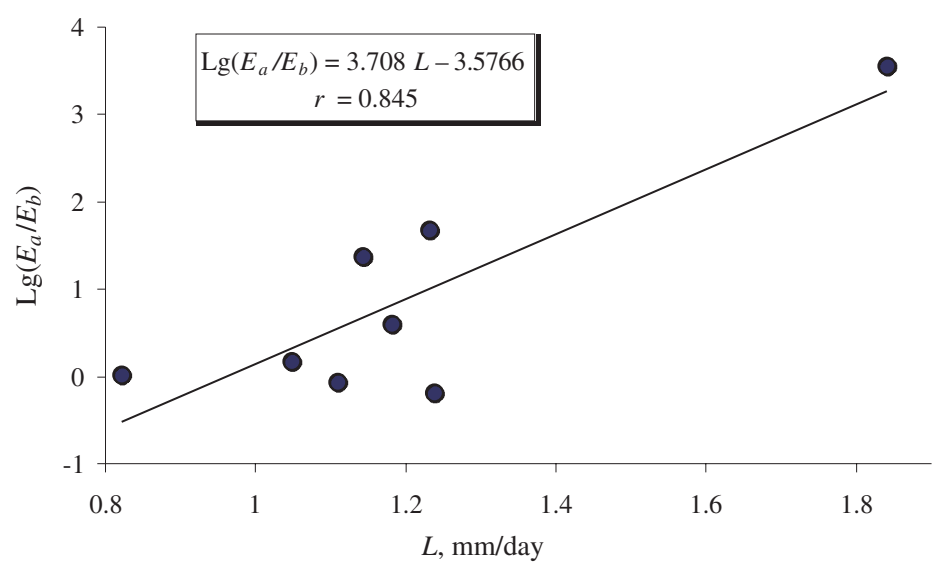

Fig. 10. Averaged annual values of $E_{a} / E_{b}$ being the ratio of total seismic energy of earthquakes of the North Tien Shan and adjacent areas occurring 5 days after and 5 days before MHD-generator start-ups in the upper 5 $\mathrm{km}$ layer plotted as function of average annual level of atmospheric precipitation $L$ in Bishkek site (dots) and fitted line. The values $E_{a} / E_{b}$ are plotted on a logarithmic scale.

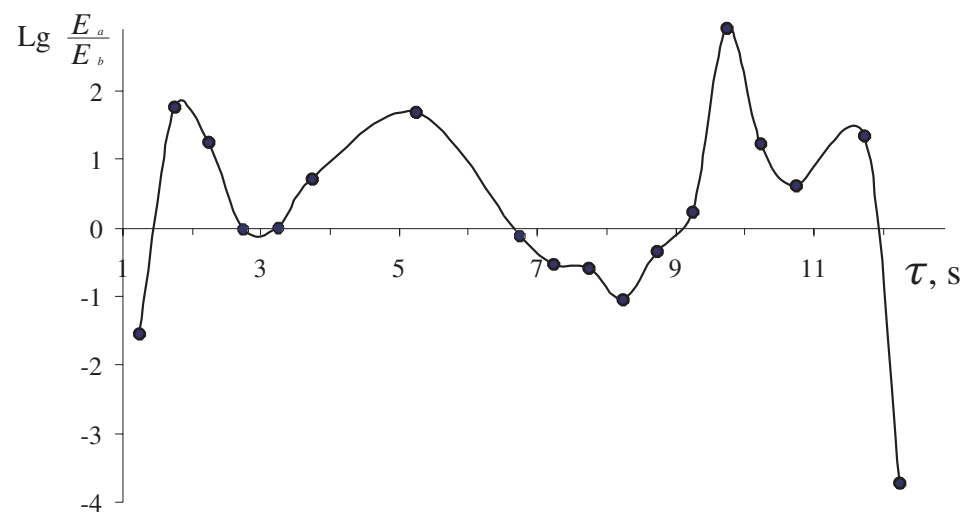

Fig. 11. Ratio $E_{a} / E_{b}$ of total seismic energy of earthquakes of the North Tien Shan and adjacent areas occurring after and before MHD-generator start-ups as a function of the electromagnetic pulses duration. The values $E_{a} / E_{b}$ are plotted on a logarithmic scale.

seismic energy of earthquakes. The 6.75-9.1 s duration pulses cause a similar effect. The high efficiency is observed for pulses of duration $\sim 2$ s, 3.5-6.7 $\mathrm{s}$ and 9.4-11.8 s. The sign-variable character of the curve suggest that layers where the electrical effects increases the flow of seismic energy and layers where the electrical impact decreases them alternate with depth.

\section{Discussion}

Changes in seismicity could be connected with the influence of some external factors. It is shown for the Central Asian region (Nikolaev and Vereshagina, 1991a,b; Tarasov and Tarasova, 1995) that $m_{b}>5$ earthquakes and underground nuclear explosions significantly influ- 
ence seismicity at a distance up to $1500 \mathrm{~km}$ The influence of geomagnetic storms on seismicity of the observation area is shown in (Tarasov and Tarasova, 2002; Sobolev and Ponomarev, 2003). Thus, activation of remote earthquakes, nuclear tests or increasing geomagnetic activity before MHD-generator startups could be the reason for the observed increasing in seismicity.

In order to check this assumption, the selection of $m_{b}>5$ earthquakes, which occurred within the time window \pm 30 days about the time of each of 114 MHD-generator runs at distances up to $1500 \mathrm{~km}$ from the center the region was made from the World Earthquake Catalogue of ISC. As in a previous study, obtained selection was used to find $N_{t}$, which is the total daily number of remote earthquakes before and after all 114 MHD-generator start-ups (fig. 12a). Estimations of the average background level and $99 \%$ confidence interval were made for all earthquakes with $m_{b}>5$, which occurred from 1983 to 1990 within the distance range of $1500 \mathrm{~km}$. In the same way we obtained daily numbers of underground nuclear explosions (fig. 12b). For analysis of geomagnetic activity we drew up the curves of changes in average daily values of $D_{s t}$ index (fig. $12 \mathrm{c}$ ) and $K_{p}$ index (fig. 12d), which were averaged for all of 114 time windows. Figure 12a-d shows that before MHD-generator start-ups $(t<0)$ there is no anomalous increase in the number of remote earthquakes or nuclear explosions, as well as anomalous changes of geomagnetic activity. Therefore the observed effect is not connected with the influence of these factors.

Some articles, for example (Oike and Yamada, 1994), detected a correlation between shallow seismicity and atmospheric thunderstorm activity. Unfortunately we have no information on thunderstorms in the region. In order to estimate this factor all MHD-generator runs were divided into two groups according to seasons: from March to August and from September to February. In the observation area, the first period is characterized by high thunderstorm activity, in the second period there are no thunderstorms. However, a significant increase in seismic activity after MHD-generator startups was observed for both groups. It means that the effect is not connected with thunderstorm activity.

One more reason for activation of seismicity could be the mechanical impact of the MHD-generator on the ground surface. Unfortunately it was impossible to check this hypothesis in this region. However, such estima-

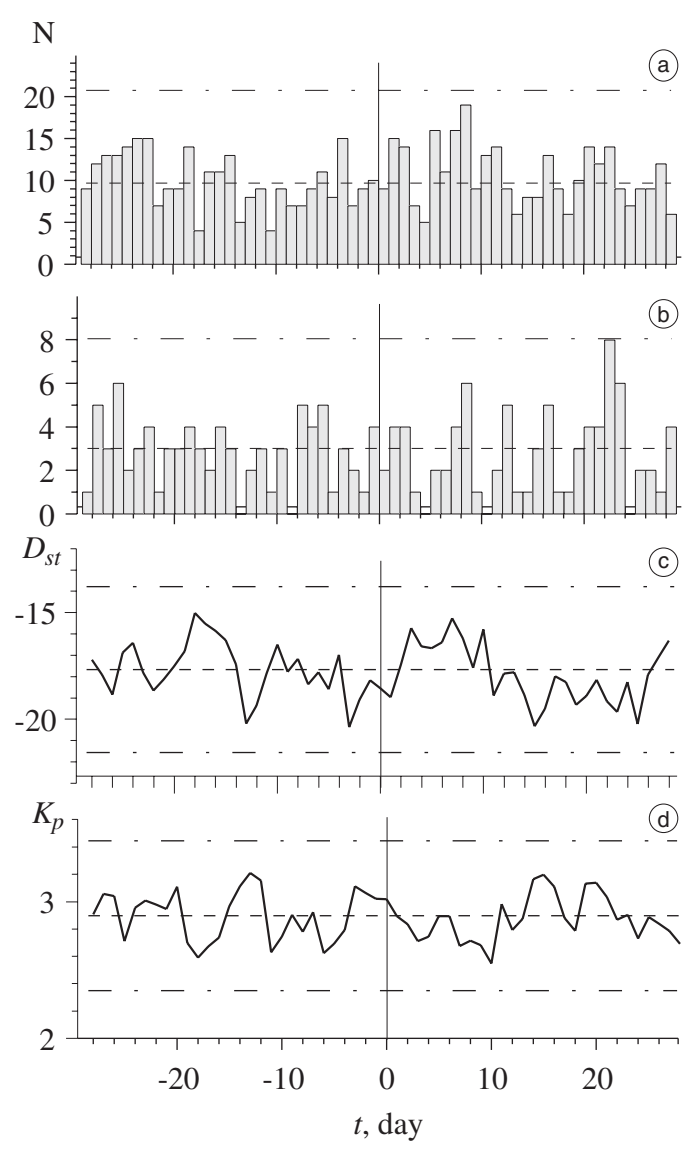

Fig. 12a-d. Daily number of $m_{b}>5$ remote earthquakes (up to $1500 \mathrm{~km}$ ) in the area (a) of the Northern Tien Shan and adjacent territories, b) of semipalatinsk underground nuclear explosions, averaged (c) $D_{s t}$ and (d) $K_{p}$ geomagnetic indices before $(t<0)$ and after $(t>0) 114$ MHD-generator runs on the Bishkek test site plotted against time (in days). The dashed lines indicate a mean background level and 99\% confidence interval. 
tion has been made for analogous experiments in Garm region of Tadjikistan (Tarasov, 1997). Along with the deep electrical sounding seismic soundings of the crust were executed by explosions of $400 \mathrm{~kg}$ of explosive (Gamburtsev et al., 1983) (within the period from 1979 to 1984 there were executed 276 explosions).

Figure 13a,b show changes in the daily number of earthquakes before and after MHDgenerator start-ups in Garm region (from Tarasov, 1997), and fig. 13c shows changes of $N_{t}$ before and after explosions in the same area. It is clearly seen that the flow of seismic events grows after the MHD-generator startups. However, unlike nuclear explosions, rela-
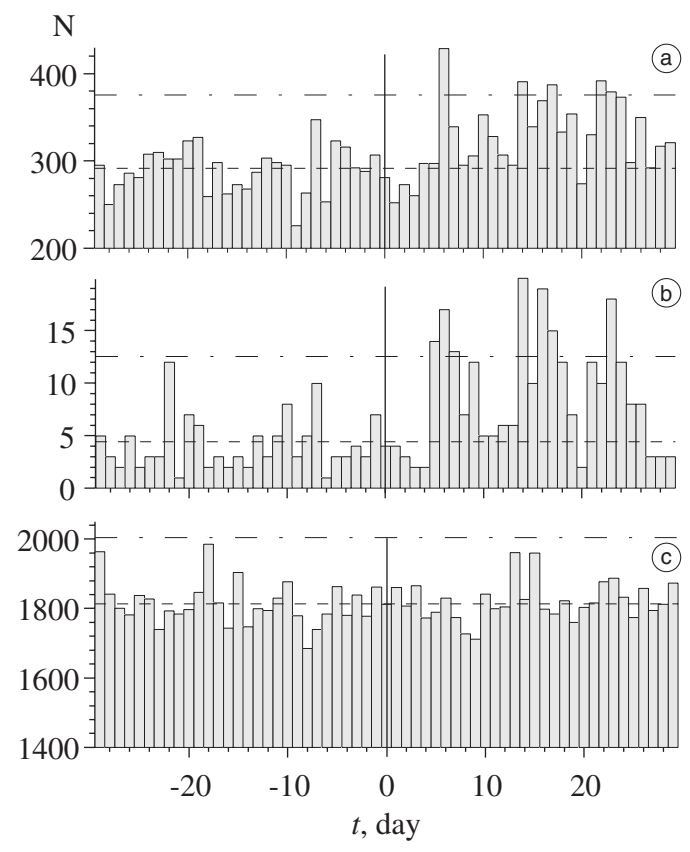

Fig. 13a-c. Daily number of earthquakes in the Garm region of Tadjikistan before $(t<0)$ and after $(t>0)$ 34 MHD-generator runs on the Garm test site plotted against time for the entire area (a) and for upper 5$\mathrm{km}$ layer of the Tadjik Depression (b), daily number of earthquakes in the same area of Tadjikistan before $(t<0)$ and after $(t>0) 276$ local chemical explosions of $400 \mathrm{~kg}$ explosive (c). The dashed lines indicate a mean background level (lower lines) and 99\% confidence interval (upper lines). tively weak chemical explosions do not cause noticeable changes in seismicity. While their impact on the ground surface is much stronger than the mechanical impact of the MHD-generator, it is possible to conclude that the observed effect is caused by its electromagnetic, but not mechanical impact.

It is interesting that in Garm region after electromagnetic impacts the most long-standing activation of seismicity was observed (fig. 13a-c), beginnning 6 days after experiments, but not after 2 days as in fig. 1a-d. Such delay of activation after the time of impact shows that electromagnetic pulses initiate some process, which causes an increase in seismicity, and the time of its development depends on rock properties. A similar dependence was observed in Garm region after remote nuclear explosions (Tarasov and Tarasova, 1995).

It is possible that rock properties determine not only the time of delay, but influence the efficiency of effect. It was shown above that in Paleozoic structures of the southern part of the region a strong activization arises after MHDgenerator runs, while in Mesozoic and Cenozoic structures of its northern part there is practically no increase in seismicity. This fact could have the another explanation. In connection with the geoelectric cross-sections of the observation area presented in Volykhin et al. (1993), it is possible to assume the existence of a high conductive layer, which plunges from the MHD-generator site to the south and is a kind of channel which directs most of the pulse energy in that direction.

However, the efficiency of the initiating effect of electromagnetic pulses is influenced by other, more complicated factors too. Periodic changes in $E_{a} / E_{b}$ with an increased distance from the MHD-generator, shown in fig. 5, could be connected with the block structure of the crust. Estimations of prevailing dimensions of the crust blocks for Tien Shan give 70 and 140 km (Sadovsky and Pisarenko, 1989), which is close enough to periods of change in $E_{a} / E_{b}$ in fig. 5. The other explanation of such periodicity could be in the long scale anticorrelation of rock properties (Sahimi et al., 1993).

At this stage, the physical mechanism of the process of initiation of seismicity by electro- 
magnetic pulses is not clear. Now it is difficult to explain with confidence the observed spatialtemporal characteristics of this effect. However, observed phenomena show new properties of triggered seismicity and could be useful for investigation of a process of earthquake initiation.

\section{Conclusions}

It has been shown that 2-6 days after effect of high energy electromagnetic pulses emitted by the MHD-generator there is activation of relatively small earthquakes in the region under study. The total seismic energy of initiated earthquakes is six orders higher than the energy transmitted by the MHD-generator to the radiating dipole.

Efficiency of the initiating effect of electromagnetic pulses depends on the natural level of seismic activity over the region, electromechanical characteristics of rocks, depth and distance to dipole.

A 5-6 years periodicity of spatial-temporal correlation of earthquake density was detected indicating the existence of periods of «fast» changes in spatial distribution of seismicity over time, which alternate every $2.5-3$ years by periods of relative stabilization. It is shown that the effect of electromagnetic pulses increases the stability of spatial distribution of seismicity over time and simultaneously speeds up cycles of its transformations, which develop on stabilization background.

The results of this study show that the effect of high energy electromagnetic pulses radiated by MHD-generators causes substantial spatial-temporal changes in the seismicity of earthquake source zones and accelerates the release of energy stored in the crust due to the activity of natural tectonic processes, thus, serving as a kind a trigger.

Based on energy balance assumption for the crust, it can be concluded that a man-made increase in part of the seismic energy radiated in the form of flow of relatively small earthquakes leads to an additional release of tectonic stresses, thereby diminishing the likelihood of catastrophic events (or at any rate, reduces the energy of such events).
If such assumption is confirmed, the detected effect could be used to develop a technique of reduction of seismic hazard by artificial discharge of tectonic stress by electromagnetic pulses.

\section{Acknowledgements}

We are grateful to Kostas Eftaxias for useful advice and discussion. This work was supported by INTAS-99-0064 grant.

\section{REFERENCES}

Chelidze, T., V. De Rubeis, T. Matcharashvili and P. Tosi (2002): Influence of strong electromagnetic discharges on the dynamics of earthquakes time distribution at the Bishkek test area (Central Asia), in Proceedings of ESC XXVIII General Assembly, Genova 2002.

Gamburtsev, A.G., N.G. Gamburtseva, S.P. StaroDUBROVSKAJA and V.N. FRIDMAN (1983): Recording waves and seismic model of the Earthcrust on the Dushanbinskiy test site, in Seismic Sounding of Source Zones, edited by A.V. NikOLAEV (Nauka, Moscow), 109-122.

MikHAilova, N.N. (1990): Catalog of Earthquakes in North Tien Shan and Adjacent Area (Nauka, AlmaAta), pp. 224.

NikolaeV, A.V. and G.M. Vereshagina (1991a): About triggering of earthquakes by earthquakes, Dokl. Acad. Nauk SSSR, 318 (2), 320-324.

Nikolaev, A.V. and G.M. Vereshagina (1991b): About triggering of earthquakes by underground nuclear explosions, Dokl. Acad. Nauk SSSR, 319 (2), 333-336.

OIKE, K. and T. YAMADA (1994): Relationship between shallow earthquakes and electromagnetic noises in LF and VLF ranges, in Electromagnetic Phenomena Related to Earthquake Prediction, edited by M. HAYAKAWA and Y. FujINAWA (Terra Scientific Publishing Co., Tokyo), 115-130.

Rautian, T.G., V.I. Khalturin, M.S. ZaKirov, A.G. Zemtsova, A.P. Proskurin, B.G. Pustovitenko, A.N. Pustovitenko, L.G. Sinelnikova, A.G. Filina and I.S. SHENGELIA (1981): Experimental Exploration of Seismic Coda (Nauka, Moscow), pp. 141.

SADOVSKY, M.A and V.F. PISARENKO (1989): Discontinuous hierarchical model of the geophysical medium, in $\mathrm{Mul}$ tidisciplinary Studies in Terrestrial Physics, edited by S. NegmatulaeV and V. Fremd (Nauka, Moscow), 9-26.

SAHimi, M., M. Robertson and C. SAmmis (1993): Fractal distribution of earthquake hypocenters and its relation to fault patterns and percolation, Phys. Rev. Lett., 70, 2186-2189.

Sobolev, G.A. and A.V. Ponomarev (2003): Physics of Earthquakes and Precursors (Nauka, Moscow), pp. 270.

TARASOV, N.T. (1997): Crustal seismicity variation under electric action, Dokl. Acad. Nauk SSSR, 353 (4), 542-545

TARASOV, N.T. and N.V. TARASOVA (1995): Response of 
seismoactive medium to nuclear explosions, in Earthquakes Induced by Underground Nuclear Explosions, edited by R. CONSOLE and A. NiKOLAEV, NATO ASI Series 2. Environment (Sprunger-Verlag, Berlin Heidelberg), 4, 215-223.

TARASOV, N.T. and N.V. TARASOVA (2002): The effect of geomagnetic storms on the seismicity, in Proceedings of Third International Workshop on Magnetic, Electric and Electromagnetic Methods in Seismology and Volcanology, Moscow, Russia, 3-6 September 2002 (Institute of Physics of the Earth, Moscow), 206-208.

Tarasov, N.T., N.V. Tarasova, A.A. Avagimov and V.A.
ZEIGARNIK (1999): The effect of high energy electromagnetic pulses on seismicity in Central Asia and Kazakhstan, Volc. Seismol., 4-5, 152-160.

Volykhin, A.M., V.D. Bragin, A.P. Zubovich, N.L. Aitov, J.P. Babakov, V.J. BataleV, A.G. BloKh, E.L. Geller, V.A. ZEIGARniK, E.I. IVANOV, J.I. IsAev, N.A. KoshKIN, R.K. Kuzmin, T.I. Kulevtsova, A.I. Matix, N.N. ORLENKo, A.V. Pisakin, A.N. Revtov, M.F. Retinsky, A.R. Rung, A.K. Ribin, B.G. TKatchenko, J.A. TrAPEZNIKOV, P.S. TuROVSKY, V.V. FinIAKIN, E.V. SHARONOVA and A.P. Shishkina (1993): Geodynamic Processes in Geophysical Fields (Nauka, Moscow), pp. 158. 